\title{
A First Assessment of the Corrections for the Consistency of the IAU2000 and IAU2006 Precession-Nutation Models
}

\author{
José M. Ferrándiz, Dhygham Al Koudsi, Alberto Escapa, Santiago Belda, \\ Sadegh Modiri, Robert Heinkelmann, and Harald Schuh
}

\begin{abstract}
The Earth precession-nutation model endorsed by resolutions of each the International Astronomical Union and the International Union of Geodesy and Geophysics is composed of two theories developed independently, namely IAU2006 precession and IAU2000A nutation. The IAU2006 precession was adopted to supersede the precession part of the IAU 2000A precession-nutation model and tried to get the new precession theory dynamically consistent with the IAU2000A nutation.

However, full consistency was not reached, and slight adjustments of the IAU2000A nutation amplitudes at the micro arcsecond level were required to ensure consistency. The first set of formulae for these corrections derived by Capitaine et al. (Astrophys 432(1):355367,2005 ), which was not included in IAU2006 but provided in some standards and software for computing nutations. Later, Escapa et al. showed that a few additional terms of the same order of magnitude have to be added to the 2005 expressions to get complete dynamical consistency between the official precession and nutation models. In 2018 Escapa and Capitaine made a joint review of the problem and proposed three alternative ways of nutation model and its parameters to achieve consistency to certain different extents, although no estimation of their respective effects could be worked out to illustrate the proposals. Here we present some preliminary results on the assessment of the effects of each of the three sets of corrections suggested by Escapa and Capitaine (Proceedings of the Journées, des Systémes de Référence et de la Rotation Terrestre: Furthering our Knowledge of Earth Rotation, Alicante, 2018) by testing them in conjunction with the conventional celestial pole offsets given in the IERS EOP14C04 time series.
\end{abstract}

\section{Keywords}

Earth orientation parameters $\cdot$ Earth rotation models $\cdot$ Earth rotation theory $\cdot$ Precessionnutation

Electronic supplementary material The online version of this chapter (https://doi.org/10.1007/1345_2020_90) contains supplementary material, which is available to authorized users.

J. M. Ferrándiz · D. Al Koudsi

UAVAC, University of Alicante, Alicante, Spain

e-mail: jm.ferrandiz@ua.es

A. Escapa

UAVAC, University of Alicante, Alicante, Spain

Department of Aerospace Engineering, University of León, León, Spain
S. Belda

UAVAC, University of Alicante, Alicante, Spain

Image Processing Laboratory (IPL) - Laboratory of Earth Observation (LEO), University of Valencia, Valencia, Spain

S. Modiri $(\bowtie) \cdot H$. Schuh

Technische Universität Berlin, Institute for Geodesy and Geoinformation Science, Berlin, Germany

GFZ German Research Centre for Geosciences, Potsdam, Germany e-mail: sadegh@gfz-potsdam.de

R. Heinkelmann

GFZ German Research Centre for Geosciences, Potsdam, Germany 


\section{Introduction}

In 2000, the Resolution B1.6 of the XXIV General Assembly (GA) of the International Astronomical Union (IAU) endorsed the IAU2000A nutation theory, which entered in force on January 1, 2003. Resolution B1 of the XXVI IAU GA held in 2006 adopted the IAU2006 precession model based on the P03 theory by Capitaine et al. (2003, 2005), following the recommendations made by the IAU Division I Working Group (WG) "On Precession and the Ecliptic" (Hilton et al. 2006). Both models were then adopted by resolutions of the International Union of Geodesy and Geophysics (IUGG) taken in 2003 and 2007. As pointed up by Escapa and Capitaine (2018a), strictly speaking Resolution B1.6 approved the "IAU 2000A precession-nutation model". However, its precession component was just a set of empirical, small corrections to the offsets and rates of its and obliquity precession. Therefore, this model was not intended to supersede the former IAU1976 precession theory (Lieske et al. 1977), and Resolution B1.6 itself encouraged the development of new expressions for precession consistent with the IAU2000A model. Both models IAU2000 and IAU2006 were required to be dynamically consistent, but before the approval of the second it was already known that they were not (Capitaine et al. 2005), but some small corrections with amplitudes of few microarcseconds ( $\mu$ as) had to be added to the nutation model. However, the WG in charge considered that nutations were out of the scope of its task, and the fact was not mentioned in the IAU resolution. Later, Escapa et al. (2014, 2016, 2017) showed that a few additional terms of the same order of magnitude have to be added to the 2005 expressions to get complete dynamical consistency between the official precession and nutation models. The issue was discussed in several occasions, particularly inside the IAU/ International Association of Geodesy (IAG) Joint Working Group on Theory of Earth rotation and validation (JWG TERV), and the main authors were invited to propose actions. Escapa and Capitaine (2018b) made a joint review of the problem and proposed three alternative ways of correcting nutations to achieve consistency to certain different extents, although no estimation of their respective effects could be worked out to illustrate the proposals. The document was subject to a wide consultation extended to all the members of the Sub-WG 1, precession and nutation, of the IAU/IAG JWG TERV, as well as to many other experts, including current and past officers of IAU and IAG. Given the short time available to take a solid decision based on the actual impact of each option, it was agreed not to propose any resolution on that direction to IAU before getting more insight into the interrelationship between precession and nutation theories and analyzing the practical implications of the different possibilities. The origin of those corrections is twofold:

1. IAU2006 included a mean constant rate for $J_{2}$, proportional to the dynamical ellipticity $H_{d}$, which is a factor of all the nutation amplitudes and the rate of the precession in the longitude of the equator, at the first order of approximation;

2. IAU2006 adopted different values than IAU2000A for other important parameters, namely the constant term $\epsilon_{0}$ of the obliquity and the longitude rate, at the reference epoch J2000.0.

To give more insight into the implications of both facts, let us recall that the IAU2000 nutation amplitude for each frequency was derived by applying the MHB2000 transfer function (Mathews et al. 2002) to multiply the corresponding rigid-Earth amplitude of REN2000 (Souchay et al. 1999). The latter amplitudes are implicitly factorized by $J_{2}$ through $H_{d}$ or the $K_{S, M}$ Kinoshita's constants (Kinoshita and Souchay 1990), and besides they depend on several circular functions of the $\epsilon_{0}$ obliquity. Therefore, the total induced variations of the non-rigid Earth amplitudes cannot be got by simply making a rescaling associated only to $J_{2}$ (Escapa et al. 2014, 2016, 2017; Escapa and Capitaine 2018a,b).

\section{$2 \quad$ Fundamentals and Methodology}

Escapa and Capitaine (2018b) cast the components of those corrections in three groups according to their origin:

(a) A geometrical effect due to the impact of the IAU2000to-IAU2006 change in the obliquity value on the projection of the CIP motion in space onto the ecliptic (i.e., nutation in longitude); it keeps unchanged the amplitudes of the IAU2000A nutation referred to the IAU 2000 ecliptic.

(b) The $J_{2}$ rate effect (a dynamical effect) due to the introduction of that rate into the IAU2000 expressions for nutation.

(c) The so-called $\triangle \mathrm{PP}$ effect (a dynamical effect) due to the IAU2000-to-IAU2006 changes of the formerly said Precession Parameters (PP).

A detailed explanation appears in that reference. 


\subsection{Equations of Models}

The three models proposed for their consideration were labeled as (a), (b) and (c), and their expressions in terms of celestial pole offsets (CPO) $d X, d Y$, are:

(a)

$-d X a=+18.8 t \sin \Omega+1.4 t \sin (2 F-2 D+2 \Omega)-0.8 t^{2} \cos \Omega$,

$-d Y a=-24.6 t \cos \Omega-1.6 t \cos (2 F-2 D+2 \Omega)-0.6 t^{2} \sin \Omega$,

(b)

$-d X b=+15.4 t \sin \Omega+1.4 t \sin (2 F-2 D+2 \Omega)-0.6 t^{2} \cos \Omega$,

$-d Y b=-25.4 t \cos \Omega-1.8 t \cos (2 F-2 D+2 \Omega)-0.3 t^{2} \sin \Omega$,

(c)

$$
\begin{aligned}
-d X c & =(-6.2+15.4 t) \sin \Omega+1.4 t \sin (2 F-2 D+2 \Omega) \\
& +\left(-0.8-0.3 t^{2}\right) \sin \Omega \\
-d Y c= & (0.8-25.4 t) \cos \Omega+(0.3-1.8 t) \cos (2 F-2 D+2 \Omega) \\
& +\left(-0.8-0.3 t^{2}\right) \sin \Omega,
\end{aligned}
$$

gathering terms with amplitudes above the $\mu$ as level.

Subindexes identify the relevant model, coefficients units are $\mu$ as, time $t$ is measured in Julian centuries since $\mathrm{J} 2000.0$ and the arguments are certain linear combinations of the Delaunay ones. In all models the dominant term is that of period 18.6years and the other arguments is semiannual. Notice that the corrections are presented with reversed sign like in Escapa et al. (2017), so that the right hand sides should be added to the CPO instead of being subtracted.

\section{$3 \quad$ Methodology}

Looking at the small magnitude of the terms, the application of any of those corrections would not likely produce a significant reduction of the WRMS (weighted root mean square) of the observed CPO series, particularly if the time t is not far from the origin J2000.0. Therefore, we decided to perform the tests with a twofold purpose:

1. Test the hypothesis of potential intercourses between the nutation corrections and the coefficients of the precession polynomials at short time intervals.

2. Checking the accuracy of the precession formulae after more than a decade, not only the effect of the corrections on the "residuals" (or unexplained by theory) CPO.
Concerning the first objective, the idea behind is that a polynomial of low degree is able of providing good approximations of long period oscillations when the time interval is short enough, but not when it exceeds certain length. Because of that, for each of the proposed correction models we computed time series of daily CPO generated from their respective formulae, and fitted to them polynomials of degrees 1 to 5 , the highest degree present in the current precession model (Belda et al. 2017a,b). We used a least squares method, either un-weighted or with weights derived from the IERS EOP14C04 series (Bizouard et al. 2019) in the usual way of most EOP data analyses (AlKoudsi 2019). Different time spans were tested, paying special attention to the period with VLBI observations available when IAU2006 was derived, presumably extended not beyond 2003.

\section{$4 \quad$ Results}

We can only present some results addressing the first of the former two purposes, due to the length constraints. The full set of results will be presented in a forthcoming paper. First we consider the time interval 1984-2003. The first numeric row of Table 1 displays the WRMS (weight root mean square) of the time series for $d X$ containing only the correction (b), which was one the preferred because it contains the full set of secular-mixed (or Poisson) terms needed to rend IAU2000 consistent with IAU2006. Next rows display the WRMS after fitting polynomials of degrees $1-5$, and the coefficients of them. It can be seen that the polynomial of degree 5 provides a very accurate approximation of the corrections values. That fact can be easily visualized in Figs. 1 and 2. The upper graphics in those figures show the correction (b) values in green together with the respective fit polynomials of degrees 1 and 5. The last polynomial almost reproduces only its low frequency variability. The respective residuals are shown in the lower graphics. Lower plot of Fig. 2 suggests that the main secularmixed term of pseudo-period 18.6 years has been almost perfectly reproduced by the polynomial, and the remaining mostly semiannual oscillation is visible in the residuals.

Table 1 Polynomial approximation of daily values of correction (b) for $d X$ in the interval 1984-2003

\begin{tabular}{llrrrrrr}
\hline Degree & WRMS & Offset & Trend & $t^{2}$ & \multicolumn{1}{l}{$t^{3}$} & $t^{4}$ & $t^{5}$ \\
\hline No fit & 0.47 & & & & & & \\
\hline 1 & 0.35 & -0.18 & 0.05 & & & & \\
\hline 2 & 0.34 & -0.17 & 0.03 & -0.00 & & & \\
\hline 3 & 0.12 & 0.08 & -0.05 & -0.06 & -0.004 & & \\
\hline 4 & 0.09 & 0.02 & -0.07 & -0.04 & -0.000 & 0.0002 & \\
\hline 5 & 0.04 & 0.01 & -0.14 & -0.04 & 0.008 & 0.0015 & 0.0000 \\
\hline
\end{tabular}



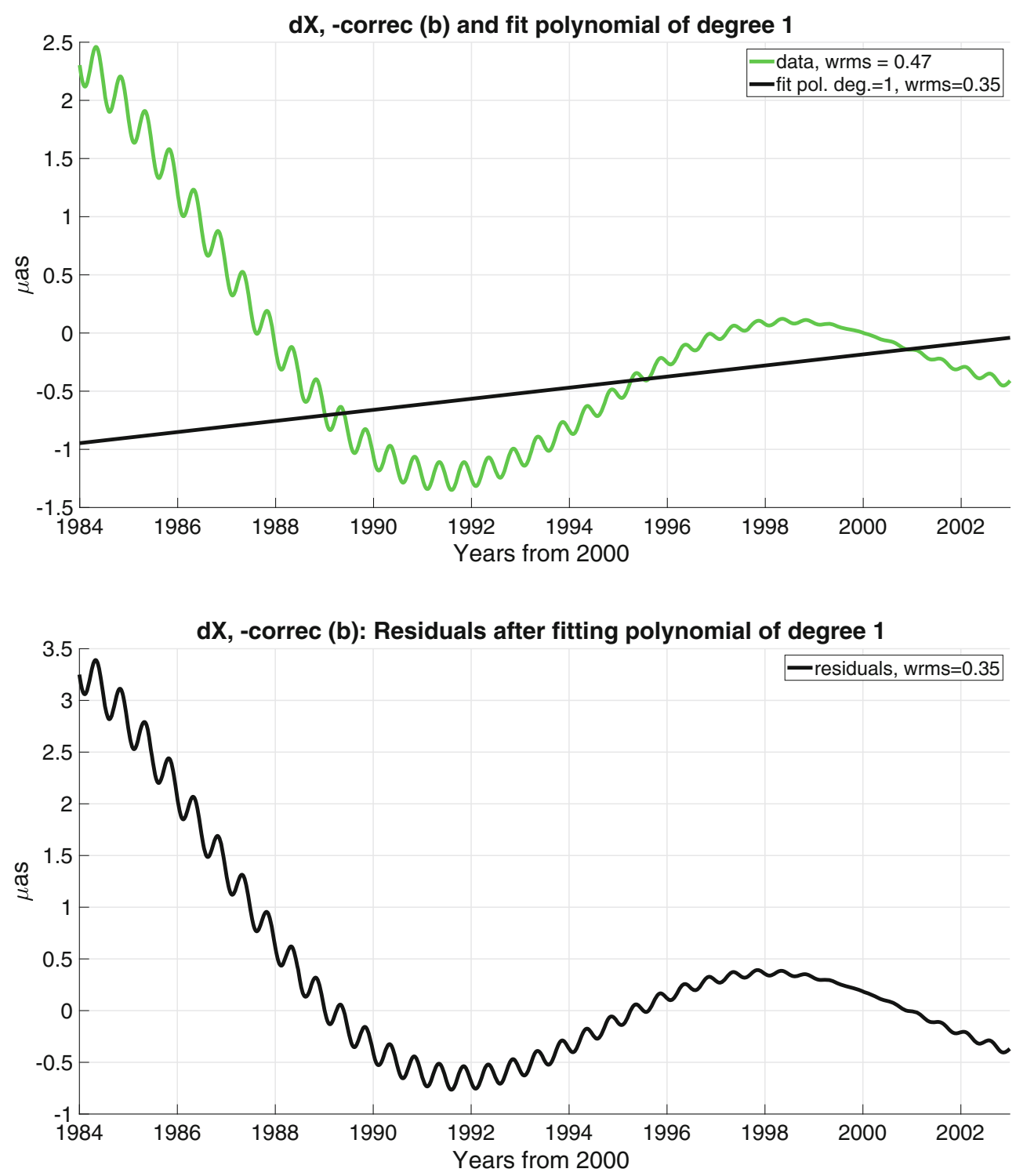

Fig. 1 Upper plot: Daily values of $d X$ correction (b) in the interval 1984-2003 and fit polynomial of degree 1. Lower plot: Residuals. Units $\mu$ as and years

Next, we present the results for $d X$ correction (b) for a much longer time interval, the two centuries 1900-2100. Table 2 shows that the signal can hardly be reproduced by any polynomial only to a minimum extent. A quick look at Fig. 3, similar to Fig. 2, allows to visualize the reason: Any low degree polynomial, even the fifth that was excellent in 1984-2003, can not reproduce the input pattern, made of many quasi-periodic cycles with amplitude increasing far from the time origin, set at year 2000 .
Finally, we present a case corresponding to a time interval ending in September 2018. Table 3 is similar to the former ones. This time the WRMS decreases, but not so much as on Table 1. Figure 4 helps to intuit why: The data curve bends too many times to be reproduced with accuracy below $1 \mu$ as by a polynomial up to degree 5 , and a long period oscillation is still visible in the residuals plot shown in the lower part of the figure. 

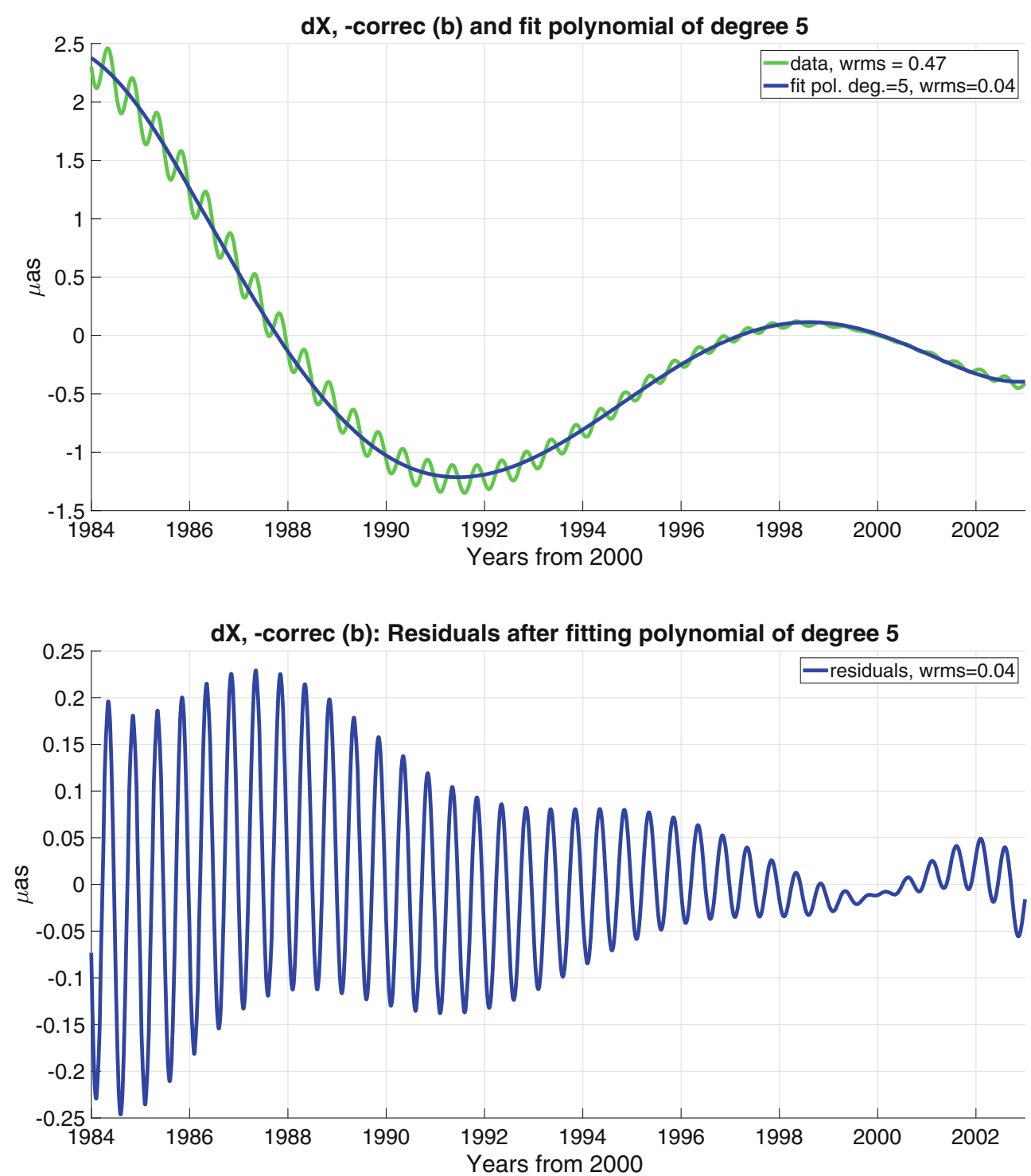

Fig. 2 Upper plot: Daily values of dX correction (b) in the interval 1984-2003 and fit polynomial of degree 5. Lower plot: Residuals. Units $\mu$ as and years

Table 2 Polynomial

approximation of daily values of correction (b) for $d X$ in the interval 1900-2100

\begin{tabular}{llrrrrrr}
\hline Degree & WRMS & Offset & Trend & $t^{2}$ & \multicolumn{1}{l}{$t^{3}$} & $t^{4}$ & $t^{5}$ \\
\hline No fit & 6.27 & & & & & & \\
\hline 1 & 6.25 & -0.20 & -0.01 & & & & \\
\hline 2 & 6.23 & 0.33 & -0.01 & -0.00 & & & \\
\hline 3 & 6.21 & 0.33 & 0.01 & -0.00 & -0.000 & & \\
\hline 4 & 6.17 & -0.47 & 0.01 & 0.00 & -0.000 & -0.0000 & \\
\hline 5 & 6.16 & -0.47 & -0.01 & 0.00 & 0.0008 & -0.0000 & -0.0000 \\
\hline
\end{tabular}

No. points $=73,050$. Units: $\mu$ as and years 

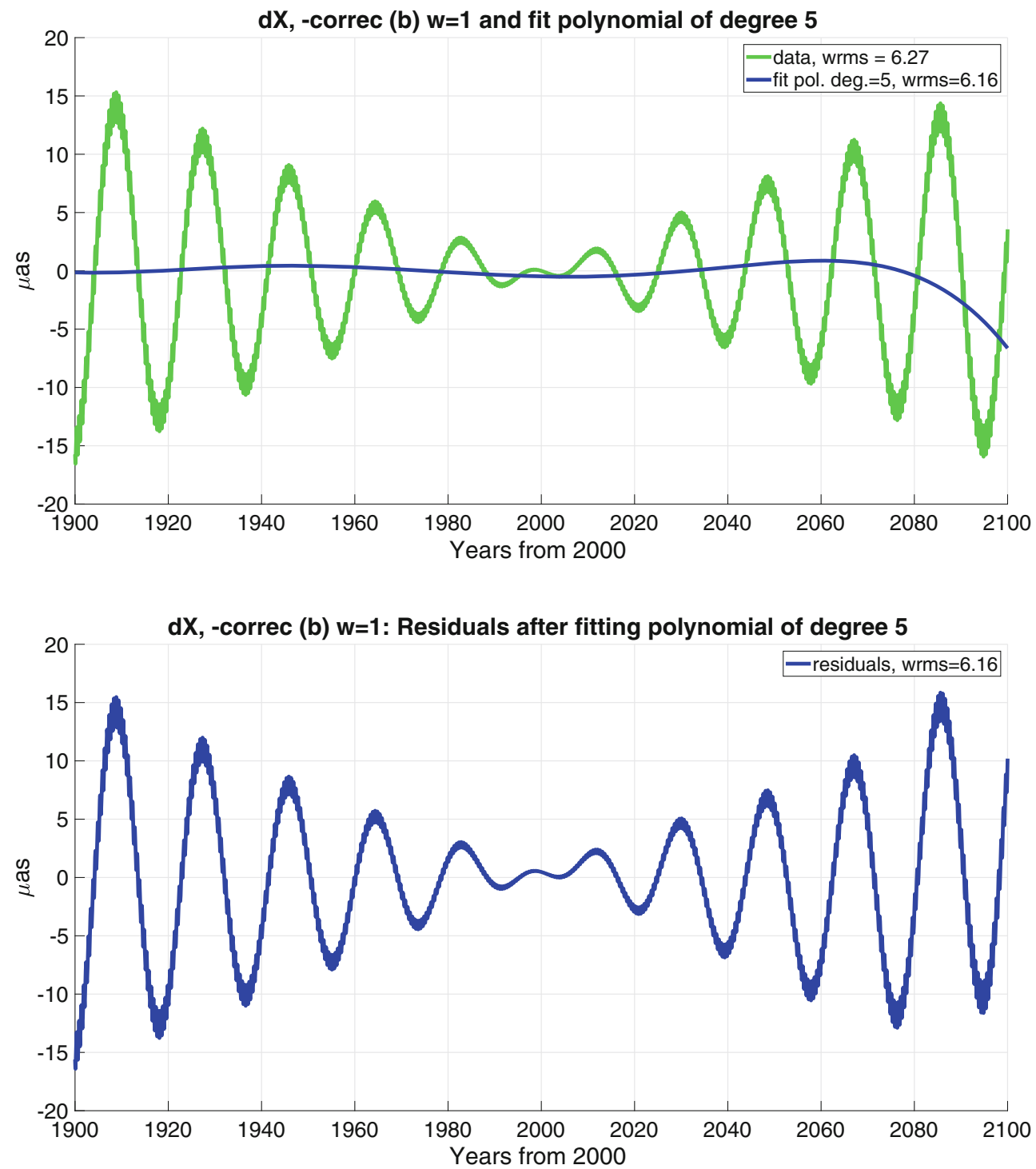

Fig. 3 Upper plot: Daily values of $d X$ correction (b) in the interval 1900-2100 and fit polynomial of degree 5. Lower plot: Residuals. Units $\mu$ as and years

Table 3 Polynomial approximation of daily values of correction (b) for $d X$ in the interval 1984-2018

\begin{tabular}{llllrlrl}
\hline Degree & WRMS & Offset & Trend & $t^{2}$ & \multicolumn{1}{l}{$t^{3}$} & \multicolumn{1}{l}{$t^{4}$} & $t^{5}$ \\
\hline No fit & 0.79 & & & & & & \\
\hline 1 & 0.76 & -0.13 & 0.03 & & & & \\
\hline 2 & 0.71 & -0.00 & 0.08 & -0.00 & & & \\
\hline 3 & 0.56 & -0.32 & 0.15 & 0.01 & -0.001 & & \\
\hline 4 & 0.45 & -0.42 & 0.06 & 0.02 & 0.000 & -0.0001 & \\
\hline 5 & 0.31 & -0.18 & 0.01 & -0.00 & 0.002 & 0.0000 & -0.0000 \\
\hline
\end{tabular}

No. points $=12,680$. Units: $\mu$ as and years 

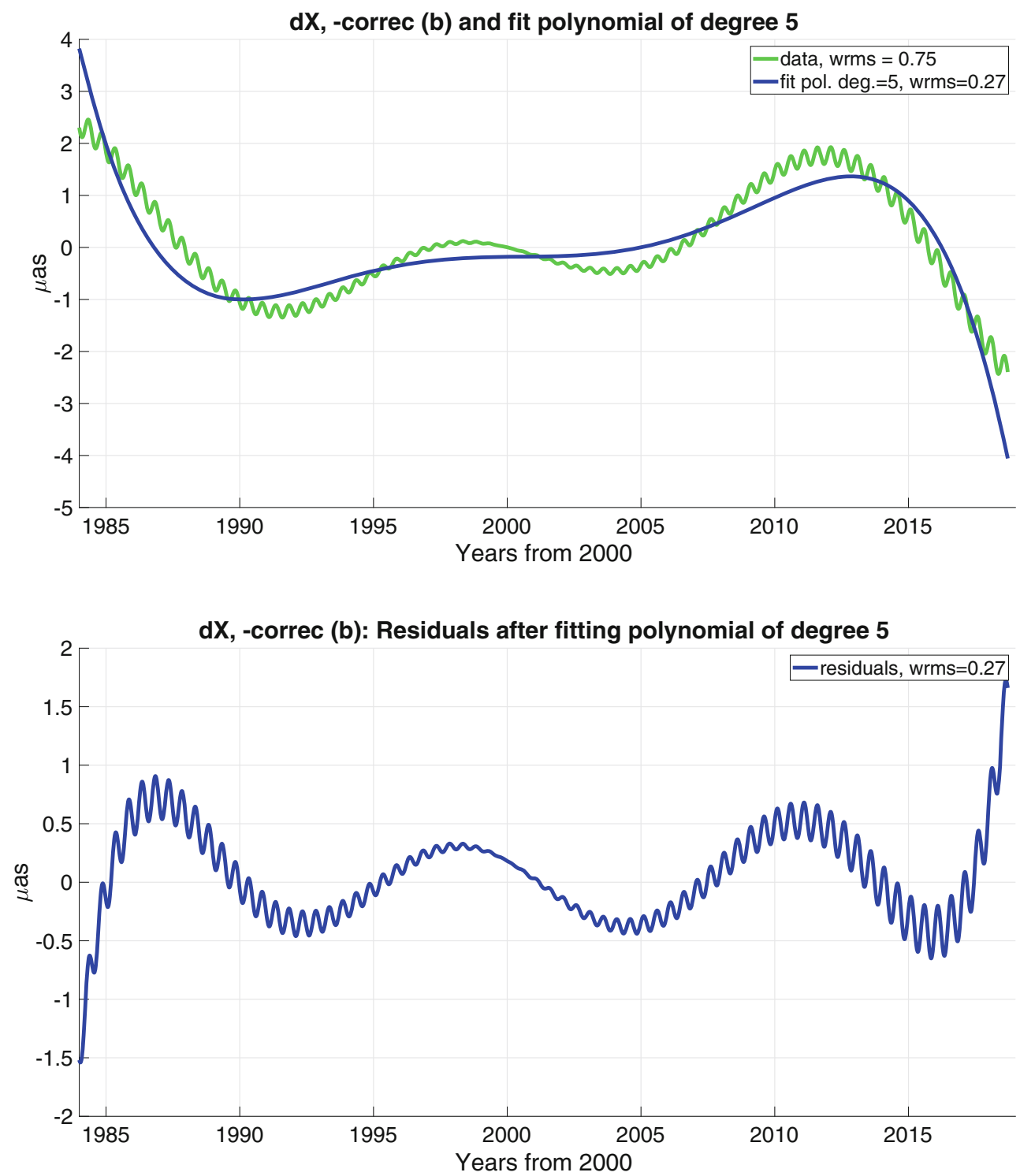

Fig. 4 Upper plot: Daily values of dX correction (b) in the interval 1984-2018 and fit polynomial of degree 5. Lower plot: Residuals. Units $\mu$ as and years

\section{$5 \quad$ Conclusions}

The results show that the lack of application of the correction making IAU2000 and IAU2006 consistent with each other can be masked in the period 1984-2003 by a fifth degree polynomial capable of absorbing more than $90 \%$ of the variance due to the additional terms that contain the nutation corrections. That fact implies that the coefficients of the IAU2006 reference polynomials include a small spurious contribution that has no physical origin but replace the effect of the absent nutation corrections.

Acknowledgement The four first authors were partially supported by Spanish Project AYA2016-79775-P (AEI/FEDER, UE).
Conflict of Interest The authors declare that they have no conflict of interest.

\section{References}

AlKoudsi D (2019) Análisis de los efectos de la insuficiente consistencia dinámica de las teorias de precesión y nutación IAU2006 e IAU2000 (Spanish language). $\mathrm{PhD}$ thesis, University of Alicante

Belda S, Heinkelmann R, Ferrándiz JM, Karbon M, Nilsson T, Schuh H (2017a) An improved empirical harmonic model of the celestial intermediate pole offsets from a global VLBI solution. Astron J 154(4): 166

Belda S, Heinkelmann R, Ferrándiz JM, Nilsson T, Schuh H (2017b) On the consistency of the current conventional EOP series and the celestial and terrestrial reference frames. J Geodesy 91(2):135-149 
Bizouard C, Lambert S, Gattano C, Becker O, Richard JY (2019) The IERS EOP 14C04 solution for Earth orientation parameters consistent with ITRF 2014. J Geodesy 93(5):621-633

Capitaine N, Wallace PT, Chapront J (2003) Expressions for IAU 2000 precession quantities. Astron Astrophys 412(2):567-586

Capitaine N, Wallace P, Chapront J (2005) Improvement of the IAU 2000 precession model. Astron Astrophys 432(1):355-367

Escapa A, Capitaine N (2018a) A global set of adjustments to make the IAU 2000a nutation consistent with the IAU 2006 precession. In: Proceedings of the Journées, des Systémes de Référence et de la Rotation Terrestre: Furthering our Knowledge of Earth Rotation, Alicante

Escapa A, Capitaine N (2018b) On the IAU 2000 nutation consistency with IAU 2006 precession,draft note. https://web.ua.es/es/wgterv/ documentos/other-documents/draft-note-escapa-capitaine-2018.pdf

Escapa A, Getino J, Ferrándiz J, Baenas T (2014) On the changes of IAU 2000 nutation theory stemming from IAU 2006 precession theory. In: Proceedings of the Journées, pp 148-151

Escapa A, Ferrándiz JM, Baenas T, Getino J, Navarro JF, BeldaPalazón S (2016) Consistency problems in the improvement of the IAU precession-nutation theories: effects of the dynamical ellipticity differences. Pure Appl Geophys 173(3):861-870
Escapa A, Getino J, Ferrándiz J, Baenas T (2017) Dynamical adjustments in IAU 2000a nutation series arising from IAU 2006 precession. Astron Astrophys 604:A92

Hilton JL, Capitaine N, Chapront J, Ferrandiz JM, Fienga A, Fukushima T, Getino J, Mathews P, Simon JL, Soffel M et al (2006) Report of the international astronomical union division i working group on precession and the ecliptic. Celest Mech Dyn Astron 94(3):351-367

Kinoshita H, Souchay J (1990) The theory of the nutation for the rigid Earth model at the second order. Celest Mech Dyn Astron 48(3):187265

Lieske J, Lederle T, Fricke W, Morando B (1977) Expressions for the precession quantities based upon the IAU/1976/system of astronomical constants. Astron Astrophys 58:1-16

Mathews PM, Herring TA, Buffett BA (2002) Modeling of nutation and precession: new nutation series for nonrigid earth and insights into the Earth's interior. J Geophys Res Solid Earth 107(B4):ETG-3

Souchay J, Loysel B, Kinoshita H, Folgueira M (1999) Corrections and new developments in rigid Earth nutation theory-III. Final tables "REN-2000" including crossed-nutation and spin-orbit coupling effects. Astron Astrophys Suppl Ser 135(1):111-131

Open Access This chapter is licensed under the terms of the Creative Commons Attribution 4.0 International License (http://creativecommons. $\mathrm{org} /$ licenses/by/4.0/), which permits use, sharing, adaptation, distribution and reproduction in any medium or format, as long as you give appropriate credit to the original author(s) and the source, provide a link to the Creative Commons licence and indicate if changes were made.

The images or other third party material in this chapter are included in the chapter's Creative Commons licence, unless indicated otherwise in a credit line to the material. If material is not included in the chapter's Creative Commons licence and your intended use is not permitted by statutory regulation or exceeds the permitted use, you will need to obtain permission directly from the copyright holder.

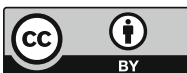

